Burmeister, O. K., Ritchie, D., Devitt, A., Chia, E., Dresser, G., \& Roberts, R. (2019). The impact of telehealth technology on user perception of wellbeing and social functioning, and the implications for service providers. Australasian Journal of Information Systems, 23. doi:10.3127/ajis.v23i0.1501

\title{
Review
}

This post publication review discusses the article by Burmeister et al. (2019) in which the authors present a study evaluating the social, economic, and wellbeing impacts of telehealth technology in the homes of community-dwelling older persons, highlighting its contribution and identifying areas for further research. The article centres on one particular project in which telehealth monitoring systems were used in homes in regional New South Wales.

The reviewed article is a timely contribution to the information systems (IS) literature. The growing number of elderly and fewer number of caregivers to meet the needs of elderly in care is a trend observed globally (Burmeister, 2016; Draper \& Sorell, 2017; Poulsen \& Burmeister, 2019). There are calls for healthcare IS interventions, such as wearable assistive devices (Fosch-Villaronga \& Özcan, 2019), telehealth (Shafiee Hanjani, Caffery, Freeman, Peeters, \& Peel, 2019), and care robots (Poulsen, Burmeister, \& Kreps, 2018; Poulsen, Burmeister, \& Tien, 2018) to help ease the impacts of this trend, particularly in regional or rural communities (Smith, Armfield, \& Caffery, 2019).

The work reviewed here creates knowledge which helps to soften the introduction of telehealth technology into the homes of the older population. Burmeister et al. (2019) further our understanding of the particular access needs of such communities, this is essential to improving user wellbeing (Pakrasi, Burmeister, Coppola, McCallum, \& Loeb, 2015), social support (Banbury et al., 2020), and health literacy (Banbury et al., 2020) with telehealth technology.

An important area highlighted in the reviewed article is good care with technology. For instance, beyond taking standard health measurements the technologies deployed in this study were adapted to obtain custom measurements related to each user's health condition. The customisation of healthcare technology demonstrates good care as it aligns with aspects of good human-delivered care which is person-centred (Abma, Molewijk, \& Widdershoven, 2009) and determined in relationship with the user in context (Upton, 2011).

Combi, Pozzani, and Pozzi (2016) highlight the lack of telehealth literature clarifying lessons learned, challenges, and recommendations related to design emerging from telehealth studies. While the authors of the reviewed article occasionally put the knowledge created throughout this study into several important learnings for future work in this area, a wider impression might have been made by setting out clear principles to guide the design, implementation, and ongoing support of telehealth technologies with older persons, much like Greenhalgh et al. (2015) have done previously. For instance, in the discussion, to mitigate economic concerns associated with telehealth the authors suggested that less expensive personal monitoring equipment alternatives "could be used in conjunction with digital scales and automatic blood pressure monitors" (Burmeister et al., 2019, p. 13), examples of alternative equipment and costs were given. As another example, in the concluding remarks, the authors noted that a nurse who assisted participants throughout the study was instrumental in improving the health literacy of participants. 
Although a variety of guiding statements, such as the two examples above, appear throughout the article reviewed, such insights ought to also have been consolidated into a set of principles for practitioners to refer to in future work and research utilizing telehealth technologies with older persons. Furthermore, these principles should link back to the research questions, chiefly promoting wellbeing, social functioning, and positive economic impacts.

On the study's aim to review social functioning impacts, additional social dimensions could have been explored to reveal further social impacts and create an extensive understanding of the telehealth-older user social ecosystem. Exemplary social dimensions include the social interactions between older persons and telehealth devices, those interactions between older users and telehealth systems capable of conversing or emotive actions, social effects on general practitioners using telehealth, social factors influencing user perceptions of telehealth technologies, and so on.

Overall, the study presented by Burmeister et al. (2019) makes invaluable contributions to the literature on telehealth and the older population. In future work, further social functioning impacts ought to be explored and the lessons learned in this study should be formulated into a set of principles for addressing the design, implementation, and ongoing support of telehealth for older persons.

\section{Adam Poulsen}

Charles Sturt University

apoulsen@csu.edu.au

\section{Author response}

The authors were given the opportunity to respond, but chose not to.

\section{References}

Abma, T., Molewijk, B., \& Widdershoven, G. (2009). Good care in ongoing dialogue. Improving the quality of care through moral deliberation and responsive evaluation. Health Care Analysis, 17, 217-235. doi:10.1007/s10728-008-0102-z.

Banbury, A., Nancarrow, S., Dart, J., Gray, L., Dodson, S., Osborne, R., \& Parkinson, L. (2020). Adding value to remote monitoring: Co-design of a health literacy intervention for older people with chronic disease delivered by telehealth - The telehealth literacy project. Patient Education and Counseling, 103(3), 597-606. doi:10.1016/j.pec.2019.10.005.

Burmeister, O. K. (2016). The development of assistive dementia technology that accounts for the values of those affected by its use. Ethics and Information Technology, 18(3), 185-198. doi:10.1007/s10676-016-9404-2.

Burmeister, O. K., Ritchie, D., Devitt, A., Chia, E., Dresser, G., \& Roberts, R. (2019). The impact of telehealth technology on user perception of wellbeing and social functioning, and the implications for service providers. Australasian Journal of Information Systems, 23. doi:10.3127/ajis.v23i0.1501. 
Combi, C., Pozzani, G., \& Pozzi, G. (2016). Telemedicine for Developing Countries. A Survey and Some Design Issues. Applied clinical informatics, 7(4), 1025-1050. doi:10.4338/ACI2016-06-R-0089.

Draper, H., \& Sorell, T. (2017). Ethical values and social care robots for older people: an international qualitative study. Ethics and Information Technology, 19(1), 49-68. doi:10.1007/s10676-016-9413-1.

Fosch-Villaronga, E., \& Özcan, B. (2019). The progressive intertwinement between design, human needs and the regulation of care technology: The case of lower-limb exoskeletons. International Journal of Social Robotics. doi:10.1007/s12369-019-00537-8.

Greenhalgh, T., Procter, R., Wherton, J., Sugarhood, P., Hinder, S., \& Rouncefield, M. (2015). What is quality in assisted living technology? The ARCHIE framework for effective telehealth and telecare services. BMC Med, 13, 91. doi:10.1186/s12916-015-0279-6.

Pakrasi, S., Burmeister, O. K., Coppola, J. F., McCallum, T. J., \& Loeb, G. (2015). Ethical telehealth design for users with dementia. Gerontechnology. Gerontechnology, 13(4), 383387. doi:10.4017/gt.2015.13.4.002.00.

Poulsen, A., \& Burmeister, O. K. (2019). Overcoming carer shortages with care robots: Dynamic value trade-offs in run-time. Australasian Journal of Information Systems, 23. doi:10.3127/ajis.v23i0.1688.

Poulsen, A., Burmeister, O. K., \& Kreps, D. (2018). The ethics of inherent trust in care robots for the elderly. In D. Kreps, C. Ess, L. Leenen, \& K. Kimppa (Eds.), This Changes Everything - ICT and Climate Change: What Can We Do? (pp. 314-328). doi:10.1007/978-3319-99605-9_24

Poulsen, A., Burmeister, O. K., \& Tien, D. (2018). A new design approach and framework for elderly care robots. Paper presented at the Australasian Conference on Information Systems, Sydney, Australia.

Shafiee Hanjani, L., Caffery, L. J., Freeman, C. R., Peeters, G., \& Peel, N. M. (2019). A scoping review of the use and impact of telehealth medication reviews. Research in Social and Administrative Pharmacy. doi:10.1016/j.sapharm.2019.12.014.

Smith, A. C., Armfield, N. R., \& Caffery, L. J. (2019). Telehealth a game changer: closing the gap in remote Aboriginal communities. Medical Journal of Australia, 211(1), 43-43.e41. doi:10.5694/mja2.50208.

Upton, H. (2011). Moral theory and theorizing in health care ethics. Ethical Theory and Moral Practice, 14(4), 431-443. doi:10.1007/s10677-011-9295-6.

Copyright: () 2020 Poulsen. This is an open-access article distributed under the terms of the Creative Commons Attribution-NonCommercial 3.0 Australia License, which permits noncommercial use, distribution, and reproduction in any medium, provided the original author and AJIS are credited. 


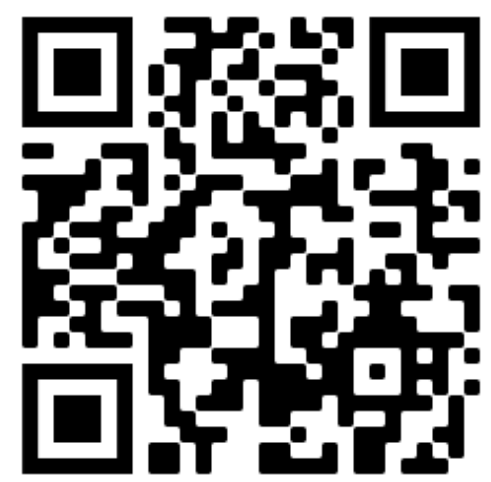

